\title{
Quality of life and informal labor among elderly persons in an intermediate Colombian city, 2012-2013
}

\section{Abstract}

Objective: Describe the quality of life of elderly informal workers in an intermediate Colombian city. Method: A descriptive study of 320 randomly selected informal workers aged older than 18 from Manizales was performed. A sub-sample of 153 people aged older than 50 , representing 47.8 percent of the study population, was performed. Two instruments were applied: one testing demographic variables and the WHOQOL-BREF quality of life questionnaire. Univariate and bivariate analyzes were performed using frequency distributions and the chi-squared test was used to identify association between variables. Results: $69.9 \%$ of the participants were men, the average age was $59.4( \pm 7.2)$ years, $62.0 \%$ had no or basic education, $52.0 \%$ belonged to social class 1 and $2 ; 6.5 \%$ had no affiliation with the health and social security system. The different dimensions of quality of life were perceived favorably, as was health. More than $50.0 \%$ were autonomous, had strong social and family networks, had not experienced negative feelings and found meaning to life. Statistical differences $(p<0.05)$ by gender and age were found for environment, sex life, daily living skills, and satisfaction with work capacity. Conclusion: The quality of life of older informal workers is well perceived and is related to a positive assessment of health. From the perspective of human development, work gives meaning to the lives of older people and encourages active, healthy and productive aging.

\footnotetext{
Universidad de Caldas, Facultad de Ciencias para la Salud, Departamento de Salud Pública. Manizales, Caldas, Colombia.

Financial support for research: Vice-Chancellor of Research and Postgraduate Studies of the University of Caldas, through the payment of interviewers, project code 0711412 .
}

Correspondence

Consuelo Vélez Alvarez

E-mail: consuelo.velez@ucaldas.edu.co
Consuelo Vélez Álvarez' María del Pilar Escobar Potes' María Eugenia Pico Merchán'

Key words: quality of life; elderly persons; aging; job satisfaction. 


\section{INTRODUCTION}

Work is considered to be a central element of social structure. As a result, it remains essential to the lives of individuals and societies, and is a vital area for the processes of socialization and personal fulfillment and the subjectivity of individuals and groups. ${ }^{1}$ It is a transformative activity which allows personal growth through physical and mental activity, particularly in terms of the eminently social conscience of an individual; both as a creator and a circulator of wealth and objects that satisfy human needs, whether tangible or intangible. ${ }^{2}$ Work can be understood as the "essential expression of a person" in contrast to approaches that make the worker an element of the production process, which result in the loss of the very essence of human actions. ${ }^{3}$

According to the Programa de Promoción de la Formalización en América Latina y el Caribe (the Program for the Promotion of Formalization in Latin America and the Caribbean) (FORLAC), the idea of informal labor corresponds to relationships of employment that are not covered by the guidelines of protection established in labor or social legislation, whether by fact or by law, and involve limited resources of capital, low productivity and a lack of labor rights. ${ }^{4}$ According to Busso, ${ }^{5}$ the most comprehensive definition is precarious labor, which covers the informal economy, informal work, unregistered work and particular or specific types of job. Salas, ${ }^{6}$ in the 1990s, proposed to abandon the idea of the informal sector, replacing it with the analysis and study of micro-units of labor. Osta ${ }^{7}$ argues that informality stems from a purely economic and structural approach, linked to the evolution of both these ideas and to unemployment, and adopts the idea of the informal economy as a complex phenomenon of nature, in which interventions should be implemented to reduce its presence and to incorporate workers and entrepreneurs into the formal sector, to improve working conditions and quality of life. According to Guerra, ${ }^{8}$ there are differences in the production types and characteristics of the situations of self-employed workers, which are dedicated to subsistence activities such as street vendors, refuse pickers, shoeshines and scrap dealers.

According to Arias \& Bernardini, ${ }^{9}$ elderly persons have a range of vulnerabilities related to health, housing, social security, education, and labor issues, among other concerns. Labor issues are particularly important, as often the elderly are marginalized in the areas of the job market through displacement by the younger population. Many find in informal work a source of income and a way of becoming active participants in their family and society, always remembering that this type of social and labor vulnerability represents a structural and economic problem.

Quality of life is generally defined as the wellbeing, happiness and satisfaction of an individual, providing a certain capacity for action, performance or positive feeling about his or her life; the World Health Organization (WHO) defines it as "individual's perception of their position in life in the context of the culture and value systems in which they live and in relation to their goals, expectations, standards and concerns". ${ }^{10}$

The term "quality of life" refers to the physical, emotional and social well- being of people and their ability to function and to perform typical activities of daily living.

Dulcey-Ruiz ${ }^{11}$ suggests that quality of life should be considered as a continuous construction throughout the life cycle of an individual, with an emphasis on aging, changing and interacting in different dimensions that affect and condition human beings. Quality of life among older people oscillates between two poles: the positive pole when networks of family and social support, health and adequate material conditions for living are in 
place; and the negative pole, when the individual becomes dependent, with functional limitations and a lack of social support networks and selfrealization. $^{12}$

With regard to productive aging in our societies, there are a variety of opinions based on the phenomenon of aging, associated with negative elements that associate it with a culturally homogeneous group characterized by inactivity, a lack of productivity, and dependency. According to Miralles, ${ }_{13}^{13}$ reductionist positions that relegate older people to a group whose needs can only be met through care, excluding their productive potential and the day-to-day support they provide to the people who share their lives and the community to which they belong and live, should be reconsidered.

The concept of productive aging is related to the idea of productivity, connected to the collective benefit that older people obtain through their own individual actions and activities, according to the Instituto de Mayores y Servicios Sociales (Institute for the Elderly and Social Services) (IMSERSO). ${ }^{14}$ While productivity is important for personal use within this concept, it is essential that it is accompanied by participation in and relationships with the social environment, and support of civic and community activities.

Elderly persons participate in a variety of occupations and work that maintains their productive capacity in everyday life to support the activities of the families and communities in which they live, or work that provides for their financial needs, such as the selling of goods in the streets and other tasks. Productive aging involves the dimensions of paid work, family-domestic work, volunteer work in the community and educational and cultural activities. ${ }^{13}$

In this context, the objective of this paper is to describe the quality of life of over 50 informal workers in an intermediate Colombian city.

\section{METHOD}

The study was conducted in Manizales, the capital of Caldas, located in central-western Colombia, which forms part of the Triángulo del Café ("the Coffee Triangle") (Manizales, Pereira and Armenia). In 2012 the city had 391,640 inhabitants, of whom 101,666 (26.0\%) were aged older than 50 years.

A descriptive study was conducted of 320 informal workers aged 18 years or over during the period 2012-2013. The subjects were randomly selected from the 1,300 informal workers in the city, after approval by the Ethics Committee of the Universidad de Caldas (the University of Caldas) and subject to the signing of a free and informed consent form (Minsalud, Colombia Resolution 08430 of 1993). This work was based on a total of 153 people aged older than 50 , who accounted for $47.8 \%$ of the total sample selected for the study. This inclusion criterion was based on Colombia's Política Nacional de Envejecimiento y Vejez 2007-2019 (the National Policy on Old Age and Aging 2007-2019), in which those aged over 50 and in situations of risk, such as informal workers, can be considered as elderly. ${ }^{15}$

Two instruments were used. One registered the sociodemographic variables of gender, age, education, social stratum (in Colombia this refers to the classification of residential property, which serves as a reference for establishing tariffs for public utilities using the categories of 1, 2 and 3 (lower); 4 (medium); and 5 (upper)), and health system affiliation (defined as linking the different modalities of receiving health care, namely contributive, unaffiliated and poor, subsidized and through private healthcare). The other instrument used was the WHOQOL-BREF quality of life questionnaire validated by $\mathrm{WHO}$, an instrument that provides a perception of quality of life in four domains: physical, psychological, interpersonal relationships and environment. It has shown good discriminant validity of content and test-retest reliability. ${ }^{16}$ 
The information was entered into Excel $^{\circledR}$ and processed using the SPSS software package version 15.0, licensed from the Universidad de Caldas (University of Caldas). Univariate and bivariate analyzes were performed using frequency distributions and the chi-squared statistical test was used to show association between variables.

\section{RESULTS}

Table 1 shows that $69.9 \%$ of participants were men, the minimum age was 50 years; the average age was $59.4( \pm 7.2)$ years; $62.0 \%$ had no educational or primary school education only; $52.3 \%$ belonged to strata 1 and 2 and $6.5 \%$ were not affiliated to the health system.

Table 1. Sociodemographic characteristics of elderly informal workers ( $N=153)$. Manizales, Caldas, 2012-2013

\begin{tabular}{c|c|c|c}
\hline Variable & Categories & $\mathrm{n}$ & $\%$ \\
\hline \multirow{2}{*}{ Gender } & Female & 46 & 30.1 \\
& Male & 107 & 69.9 \\
\hline \multirow{3}{*}{ Age } & $50-59$ & 88 & 57.5 \\
& $60-69$ & 46 & 30.1 \\
& 70 or older & 19 & 12.4 \\
\hline \multirow{5}{*}{ Education } & No education & 14 & 9.2 \\
& Primary education & 81 & 52.9 \\
& Secondary education & 55 & 35.9 \\
& University education & 1 & 0.6 \\
& Other & 2 & 1.3 \\
\hline \multirow{5}{*}{ Social Stratum } & 1 & 37 & 24.2 \\
& 2 & 43 & 28.1 \\
& 3 & 60 & 39.2 \\
& Contributive & 10 & 6.5 \\
& None & 3 & 2.0 \\
\hline \multirow{5}{*}{ Healthcare regime } & Special scheme & 10 & 33.3 \\
& Subsidized & 3 & 6.5 \\
& & 89 & 2.0 \\
& & & 58.2 \\
\hline
\end{tabular}


Time spent working informally varied between one and 59 years with an average of $23( \pm 13)$ years; weekly income was between $\$ 4,000$ and $\$ 700,000$ Colombian pesos, averaging $\$ 92,000( \pm \$ 93,000)$ Colombian pesos.

Regarding social security, $58.2 \%$ were affiliated to the subsidized healthcare system (where the state guarantees the right to health care through a subsidy provided to the poorest members of society who do not have the ability to pay). Notably, 99.3\% were not affiliated to an Office of Risk Management (ORM). A total of $77.8 \%$ did not have a pension plan; although $83.0 \%$ owned their own business.

Quality of life was perceived as positive across the different dimensions by the informal workers aged over 50 . It is notable that $51.0 \%$ rated their health as good to excellent; consistent with the $42.0 \%$ who were satisfied with their health. In terms of quality of life, $72.5 \%$ rated it between normal and good.
A total of $60.0 \%$ said that pain (physical) either did not hinder or hindered them only a little from doing what they needed to do in day to day life; $47.0 \%$ did not need medical treatment to function in their daily lives; $77.0 \%$ were able to travel from one place to another; $52.0 \%$ were satisfied and very satisfied with their sleep; $58.0 \%$ were satisfied with their ability to perform activities of daily living and $55.0 \%$ were happy with their work (table 2); $82.0 \%$ enjoyed life, either to a regular degree or extremely, and $43.0 \%$ said life makes sense; $75.0 \%$ accepted their physical appearance; $46.0 \%$ said they never or rarely had negative feelings, such as sadness, hopelessness, anxiety or boredom (Table 3)

Table 4 shows the characteristics relating to the dimension of surrounding environment, which was considered by $45.0 \%$ of participants to be only a little healthy or unhealthy; $69.0 \%$ did not have enough or had little money to meet their needs; $58.0 \%$ had little or no opportunity for recreation, relaxation or fun; $49.0 \%$ were not satisfied with health services. 
Table 2. Characteristics of the sample ( $N=153)$ related to physical dimension.Manizales, Caldas, 2012-2013.

To what extent do you feel that physical pain prevents you from doing what you need to do?

\begin{tabular}{c|c|c|c|c|c|c|c|c|c}
\hline \multicolumn{2}{c|}{ Not at all } & \multicolumn{2}{c|}{ A little } & \multicolumn{2}{c|}{$\begin{array}{c}\text { A moderate } \\
\text { amount }\end{array}$} & \multicolumn{2}{c}{ Very much } & \multicolumn{2}{c}{$\begin{array}{c}\text { An extreme } \\
\text { amount }\end{array}$} \\
\hline $\mathrm{n}$ & $\%$ & $\mathrm{n}$ & $\%$ & $\mathrm{n}$ & $\%$ & $\mathrm{n}$ & $\%$ & $\mathrm{n}$ & $\%$ \\
\hline 64 & 41.8 & 29 & 19.0 & 13 & 8.5 & 36 & 23.5 & 11 & 7.2 \\
\hline
\end{tabular}

How much do you need any medical treatment to function in your daily life?

\begin{tabular}{c|c|c|c|c|c|c|c|c|c}
\hline \multicolumn{2}{c|}{ Not at all } & \multicolumn{2}{c|}{ A little } & \multicolumn{2}{c|}{$\begin{array}{c}\text { A moderate } \\
\text { amount }\end{array}$} & \multicolumn{2}{c}{$\begin{array}{c}\text { Very much } \\
\text { An extreme } \\
\text { amount }\end{array}$} \\
\hline $\mathrm{n}$ & $\%$ & $\mathrm{n}$ & $\%$ & $\mathrm{n}$ & $\%$ & $\mathrm{n}$ & $\%$ & $\mathrm{n}$ & $\%$ \\
\hline 60 & 39.2 & 13 & 8.5 & 13 & 8.5 & 49 & 32.0 & 18 & 11.8 \\
\hline
\end{tabular}

How well are you able to get around?

\begin{tabular}{c|c|c|c|c|c|c|c|c|c}
\hline \multicolumn{2}{c|}{ Very poor } & \multicolumn{2}{c|}{ Poor } & \multicolumn{2}{c|}{$\begin{array}{c}\text { Neither poor nor } \\
\text { good }\end{array}$} & \multicolumn{3}{c}{ Good } & \multicolumn{3}{c}{ Very good } \\
\hline $\mathrm{N}$ & $\%$ & $\mathrm{~N}$ & $\%$ & $\mathrm{n}$ & $\%$ & $\mathrm{n}$ & $\%$ & $\mathrm{~N}$ & $\%$ \\
\hline 2 & 1.3 & 16 & 10.5 & 17 & 11.1 & 26 & 17.0 & 92 & 60.1 \\
\hline
\end{tabular}

How satisfied are you with your sleep?

\begin{tabular}{c|c|c|c|c|c|c|c|c|c}
\hline \multicolumn{2}{c|}{ Very dissatisfied } & \multicolumn{2}{|c|}{ Dissatisfied } & \multicolumn{2}{c|}{$\begin{array}{c}\text { Neither satisfied } \\
\text { nor dissatisfied }\end{array}$} & \multicolumn{3}{c}{ Satisfied } & \multicolumn{3}{c}{ Very satisfied } \\
\hline $\mathrm{N}$ & $\%$ & $\mathrm{n}$ & $\%$ & $\mathrm{n}$ & $\%$ & $\mathrm{~N}$ & $\%$ & $\mathrm{~N}$ & $\%$ \\
\hline 13 & 8.5 & 28 & 18.3 & 32 & 20.9 & 51 & 33.3 & 29 & 19.0 \\
\hline
\end{tabular}

How satisfied are you with your ability to perform your daily living activities?

\begin{tabular}{c|c|c|c|c|c|c|c|c|c}
\hline \multicolumn{2}{c|}{ Very dissatisfied } & \multicolumn{2}{|c|}{ Dissatisfied } & \multicolumn{2}{c|}{$\begin{array}{c}\text { Neither satisfied } \\
\text { nor dissatisfied }\end{array}$} & \multicolumn{2}{c}{ Satisfied } & \multicolumn{3}{c}{ Very satisfied } \\
\hline $\mathrm{n}$ & $\%$ & $\mathrm{n}$ & $\%$ & $\mathrm{n}$ & $\%$ & $\mathrm{n}$ & $\%$ & $\mathrm{~N}$ & $\%$ \\
\hline 6 & 3.9 & 14 & 9.2 & 43 & 28.1 & 74 & 48.4 & 16 & 10.5 \\
\hline
\end{tabular}

How satisfied are you with your capacity to work?

\begin{tabular}{c|c|c|c|c|c|c|c|c|c}
\hline \multicolumn{2}{c|}{ Very dissatisfied } & \multicolumn{2}{|c|}{ Dissatisfied } & \multicolumn{2}{c|}{$\begin{array}{c}\text { Neither satisfied } \\
\text { nor dissatisfied }\end{array}$} & \multicolumn{3}{c}{ Satisfied } & \multicolumn{3}{c}{ Very satisfied } \\
\hline $\mathrm{n}$ & $\%$ & $\mathrm{~N}$ & $\%$ & $\mathrm{n}$ & $\%$ & $\mathrm{n}$ & $\%$ & $\mathrm{n}$ & $\%$ \\
\hline 3 & 2.0 & 20 & 13.1 & 45 & 29.4 & 67 & 43.8 & 18 & 11.8 \\
\hline
\end{tabular}


Table 3. Characteristics of the sample $(\mathrm{N}=153)$ related to physiological dimension. Manizales, Caldas, 2012-2013.

\begin{tabular}{|c|c|c|c|c|c|c|c|c|c|}
\hline \multicolumn{10}{|c|}{ How much do you enjoy life? } \\
\hline \multicolumn{2}{|c|}{ Not at all } & \multicolumn{2}{|c|}{ A little } & \multicolumn{2}{|c|}{$\begin{array}{l}\text { A moderate } \\
\text { amount }\end{array}$} & \multicolumn{2}{|c|}{ Very much } & \multicolumn{2}{|c|}{ An extreme amount } \\
\hline $\mathrm{n}$ & $\%$ & $\mathrm{n}$ & $\%$ & $\mathrm{n}$ & $\%$ & $\mathrm{n}$ & $\%$ & $\mathrm{n}$ & $\%$ \\
\hline 21 & 13.7 & 23 & 15.0 & 73 & 47.7 & 19 & 12.4 & 17 & 11.1 \\
\hline
\end{tabular}

To what extent do you feel your life to be meaningful?

\begin{tabular}{c|c|c|c|c|c|c|c|c|c}
\hline \multicolumn{2}{c|}{ Not at all } & \multicolumn{2}{c|}{ A little } & \multicolumn{2}{c|}{$\begin{array}{c}\text { A moderate } \\
\text { amount }\end{array}$} & \multicolumn{2}{c|}{ Very much } & \multicolumn{2}{c}{ An extreme amount } \\
\hline $\mathrm{n}$ & $\%$ & $\mathrm{n}$ & $\%$ & $\mathrm{n}$ & $\%$ & $\mathrm{n}$ & $\%$ & $\mathrm{n}$ & $\%$ \\
\hline 4 & 2.6 & 6 & 3.9 & 77 & 50.3 & 41 & 26.8 & 25 & 16.3 \\
\hline
\end{tabular}

How well are you able to concentrate?

\begin{tabular}{c|c|c|c|c|c|c|c|c|c}
\hline \multicolumn{2}{c|}{ Not at all } & \multicolumn{2}{c|}{ A little } & \multicolumn{2}{c|}{$\begin{array}{c}\text { A moderate } \\
\text { amount }\end{array}$} & \multicolumn{2}{c}{ Very much } & \multicolumn{2}{c}{ Extremely } \\
\hline $\mathrm{n}$ & $\%$ & $\mathrm{n}$ & $\%$ & $\mathrm{n}$ & $\%$ & $\mathrm{n}$ & $\%$ & $\mathrm{n}$ & $\%$ \\
\hline 6 & 3.9 & 19 & 12.4 & 45 & 29.4 & 69 & 45.1 & 14 & 9.2 \\
\hline
\end{tabular}

Are you able to accept your bodily appearance?

\begin{tabular}{c|c|c|c|c|c|c|c|c|c}
\hline \multicolumn{2}{c|}{ Not at all } & \multicolumn{2}{c|}{ A little } & \multicolumn{2}{c|}{ Moderately } & \multicolumn{2}{c|}{ Mostly } & \multicolumn{2}{c}{ Completely } \\
\hline $\mathrm{n}$ & $\%$ & $\mathrm{n}$ & $\%$ & $\mathrm{n}$ & $\%$ & $\mathrm{n}$ & $\%$ & $\mathrm{n}$ & $\%$ \\
\hline 2 & 1.3 & 9 & 5.9 & 26 & 17.0 & 84 & 54.9 & 32 & 20.9 \\
\hline
\end{tabular}

How often do you have negative feelings such as blue mood, despair, anxiety, depression?

\begin{tabular}{c|c|c|c|c|c|c|c}
\hline \multicolumn{2}{c|}{ Never } & \multicolumn{2}{c|}{ Seldom } & \multicolumn{2}{c|}{ Quite often } & \multicolumn{2}{c}{ Frequently } \\
\hline $\mathrm{n}$ & $\%$ & $\mathrm{n}$ & $\%$ & $\mathrm{n}$ & $\%$ & $\mathrm{n}$ & $\%$ \\
\hline 34 & 22.2 & 37 & 24.2 & 40 & 26.1 & 27 & 17.6 \\
\hline
\end{tabular}

How satisfied are you with yourself?

\begin{tabular}{|c|c|c|c|c|c|c|c|c|c|}
\hline \multicolumn{2}{|c|}{$\begin{array}{c}\text { Very } \\
\text { dissatisfied }\end{array}$} & \multicolumn{2}{|c|}{ Dissatisfied } & \multicolumn{2}{|c|}{$\begin{array}{l}\text { Neither satisfied } \\
\text { nor dissatisfied }\end{array}$} & \multicolumn{2}{|c|}{ Satisfied } & \multicolumn{2}{|c|}{ Very satisfied } \\
\hline $\mathrm{n}$ & $\%$ & $\mathrm{n}$ & $\%$ & $\mathrm{~N}$ & $\%$ & $\mathrm{n}$ & $\%$ & $\mathrm{n}$ & $\%$ \\
\hline 3 & 2.0 & 5 & 3.3 & 49 & 32.0 & 69 & 45.1 & 27 & 16.6 \\
\hline
\end{tabular}


Table 4. Characteristics of the sample $(\mathrm{N}=153)$ related to the environmental dimension. Manizales, Caldas, 2012-2013.

How safe do you feel in your daily life?

\begin{tabular}{c|c|c|c|c|c|c|c|c|c}
\hline \multicolumn{2}{c|}{ Not at all } & \multicolumn{2}{c|}{ A little } & \multicolumn{2}{c|}{ A moderate amount } & \multicolumn{2}{c}{ Very much } & \multicolumn{2}{c}{ Extremely } \\
\hline $\mathrm{n}$ & $\%$ & $\mathrm{n}$ & $\%$ & $\mathrm{n}$ & $\%$ & $\mathrm{n}$ & $\%$ & $\mathrm{n}$ & $\%$ \\
\hline 12 & 7.8 & 28 & 18.3 & 58 & 37.9 & 49 & 32.0 & 6 & 3.9 \\
\hline
\end{tabular}

How healthy is your physical environment?

\begin{tabular}{c|c|c|c|c|c|c|c|c|c}
\hline \multicolumn{2}{c|}{ Not at all } & \multicolumn{2}{c|}{ A little } & \multicolumn{2}{c|}{ A moderate amount } & \multicolumn{2}{c|}{ Very much } & \multicolumn{2}{c}{ Extremely } \\
\hline $\mathrm{n}$ & $\%$ & $\mathrm{n}$ & $\%$ & $\mathrm{n}$ & $\%$ & $\mathrm{n}$ & $\%$ & $\mathrm{n}$ & $\%$ \\
\hline 16 & 10.5 & 54 & 35.3 & 53 & 34.6 & 23 & 15.0 & 7 & 4.6 \\
\hline
\end{tabular}

Have you enough money to meet your needs?

\begin{tabular}{c|c|c|c|c|c|c|c|c|c}
\hline \multicolumn{2}{c|}{ Not at all } & \multicolumn{2}{c|}{ A little } & \multicolumn{2}{c|}{ Moderately } & \multicolumn{2}{c}{ Mostly } & \multicolumn{2}{c}{ Completely } \\
\hline $\mathrm{n}$ & $\%$ & $\mathrm{~N}$ & $\%$ & $\mathrm{n}$ & $\%$ & $\mathrm{~N}$ & $\%$ & $\mathrm{~N}$ & $\%$ \\
\hline 26 & 17.0 & 80 & 53.2 & 40 & 26.1 & 4 & 2.6 & 3 & 2.0 \\
\hline
\end{tabular}

How available to you is the information you need in your day-to-day life?

\begin{tabular}{c|c|c|c|c|c|c|c|c|c}
\hline \multicolumn{2}{c|}{ Not at all } & \multicolumn{2}{c|}{ A little } & \multicolumn{2}{c|}{ Moderately } & \multicolumn{2}{c|}{ Mostly } & \multicolumn{2}{c}{ Completely } \\
\hline $\mathrm{n}$ & $\%$ & $\mathrm{n}$ & $\%$ & $\mathrm{n}$ & $\%$ & $\mathrm{n}$ & $\%$ & $\mathrm{~N}$ & $\%$ \\
\hline 17 & 11.1 & 16 & 10.5 & 52 & 34.0 & 54 & 35.3 & 14 & 9.2 \\
\hline
\end{tabular}

To what extent do you have the opportunity for leisure activities?

\begin{tabular}{c|c|c|c|c|c|c|c|c|c}
\hline \multicolumn{2}{c|}{ Not at all } & \multicolumn{2}{c|}{ A little } & \multicolumn{2}{c|}{ Moderately } & \multicolumn{2}{c|}{ Mostly } & \multicolumn{2}{c}{ Completely } \\
\hline $\mathrm{n}$ & $\%$ & $\mathrm{n}$ & $\%$ & $\mathrm{n}$ & $\%$ & $\mathrm{n}$ & $\%$ & $\mathrm{~N}$ & $\%$ \\
\hline 48 & 31.4 & 42 & 27.5 & 39 & 25.5 & 20 & 13.1 & 4 & 2.6 \\
\hline
\end{tabular}

How satisfied are you with the conditions of your living place?

\begin{tabular}{|c|c|c|c|c|c|c|c|c|c|}
\hline \multicolumn{2}{|c|}{$\begin{array}{c}\text { Very } \\
\text { dissatisfied }\end{array}$} & \multicolumn{2}{|c|}{ Dissatisfied } & \multicolumn{2}{|c|}{$\begin{array}{c}\text { Neither satisfied nor } \\
\text { dissatisfied }\end{array}$} & \multicolumn{2}{|c|}{ Satisfied } & \multicolumn{2}{|c|}{ Very satisfied } \\
\hline $\mathrm{n}$ & $\%$ & $\mathrm{n}$ & $\%$ & $\mathrm{n}$ & $\%$ & $\mathrm{n}$ & $\%$ & $\mathrm{~N}$ & $\%$ \\
\hline 3 & 2.0 & 18 & 11.8 & 38 & 24.8 & 73 & 47.7 & 21 & 13.7 \\
\hline
\end{tabular}

How satisfied are you with your access to health services?

\begin{tabular}{|c|c|c|c|c|c|c|c|c|c|}
\hline \multicolumn{2}{|c|}{$\begin{array}{c}\text { Very } \\
\text { dissatisfied }\end{array}$} & \multicolumn{2}{|c|}{ Dissatisfied } & \multicolumn{2}{|c|}{$\begin{array}{l}\text { Neither satisfied nor } \\
\text { dissatisfied }\end{array}$} & \multicolumn{2}{|c|}{ Satisfied } & \multicolumn{2}{|c|}{ Very satisfied } \\
\hline $\mathrm{n}$ & $\%$ & $\mathrm{n}$ & $\%$ & $\mathrm{n}$ & $\%$ & $\mathrm{n}$ & $\%$ & $\mathrm{n}$ & $\%$ \\
\hline 46 & 30.1 & 30 & 19.6 & 36 & 23.5 & 31 & 20.3 & 10 & 6.5 \\
\hline
\end{tabular}

How satisfied are you with your transport?

\begin{tabular}{|c|c|c|c|c|c|c|c|c|c|}
\hline \multicolumn{2}{|c|}{$\begin{array}{c}\text { Very } \\
\text { dissatisfied }\end{array}$} & \multicolumn{2}{|c|}{ Dissatisfied } & \multicolumn{2}{|c|}{$\begin{array}{c}\text { Neither satisfied nor } \\
\text { dissatisfied }\end{array}$} & \multicolumn{2}{|c|}{ Satisfied } & \multicolumn{2}{|c|}{ Very satisfied } \\
\hline $\mathrm{n}$ & $\%$ & $\mathrm{n}$ & $\%$ & $\mathrm{n}$ & $\%$ & $\mathrm{n}$ & $\%$ & $\mathrm{n}$ & $\%$ \\
\hline 9 & 5.9 & 14 & 9.2 & 56 & 36.6 & 58 & 37.9 & 16 & 10.5 \\
\hline
\end{tabular}


Table 5, which corresponds to the dimension of interpersonal relationships, found that $65.0 \%$ were very satisfied with their personal relationships and $45.0 \%$ were very satisfied with the support of friends. It is noteworthy that the item with the lowest positive assessment in this dimension was satisfaction with sex life.

Table 5. Characteristics of the sample $(\mathrm{N}=153)$ related to the interpersonal dimension. Manizales, Caldas, 2012-2013.

\begin{tabular}{c|c|c|c|c|c|c|c|c|c}
\hline \multicolumn{2}{l}{ How satisfied are you with your personal relationships? } \\
\multicolumn{2}{c|}{ Very dissatisfied } & \multicolumn{2}{c|}{ Dissatisfied } & \multicolumn{2}{c|}{$\begin{array}{c}\text { Neither satisfied } \\
\text { nor dissatisfied }\end{array}$} & \multicolumn{2}{c}{ Satisfied } & \multicolumn{2}{c}{ Very satisfied } \\
\hline $\mathrm{n}$ & $\%$ & $\mathrm{n}$ & $\%$ & $\mathrm{n}$ & $\%$ & $\mathrm{n}$ & $\%$ & $\mathrm{n}$ & $\%$ \\
\hline 2 & 1.3 & 13 & 8.5 & 38 & 24.8 & 75 & 49.0 & 25 & 16.3 \\
\hline
\end{tabular}

How satisfied are you with your sex life?

\begin{tabular}{c|c|c|c|c|c|c|c|c|c}
\hline \multicolumn{2}{c|}{ Very dissatisfied } & \multicolumn{2}{c|}{ Dissatisfied } & \multicolumn{2}{c|}{$\begin{array}{c}\text { Neither satisfied } \\
\text { nor dissatisfied }\end{array}$} & \multicolumn{2}{c}{ Satisfied } & \multicolumn{2}{c}{ Very satisfied } \\
\hline $\mathrm{n}$ & $\%$ & $\mathrm{n}$ & $\%$ & $\mathrm{n}$ & $\%$ & $\mathrm{n}$ & $\%$ & $\mathrm{n}$ & $\%$ \\
\hline 22 & 14.4 & 16 & 10.5 & 62 & 40.5 & 40 & 26.1 & 13 & 8.5 \\
\hline
\end{tabular}

How satisfied are you with the support you get from your friends?

\begin{tabular}{c|c|c|c|c|c|c|c|c|c}
\hline \multicolumn{2}{c|}{ Very dissatisfied } & \multicolumn{2}{c|}{ Dissatisfied } & \multicolumn{2}{c|}{$\begin{array}{c}\text { Neither satisfied } \\
\text { nor dissatisfied }\end{array}$} & \multicolumn{2}{c}{ Satisfied } & \multicolumn{2}{c}{ Very satisfied } \\
\hline $\mathrm{n}$ & $\%$ & $\mathrm{n}$ & $\%$ & $\mathrm{n}$ & $\%$ & $\mathrm{n}$ & $\%$ & $\mathrm{~N}$ & $\%$ \\
\hline 12 & 7.8 & 27 & 17.6 & 44 & 28.8 & 53 & 34.6 & 17 & 12.1 \\
\hline
\end{tabular}

There were statistically significant differences by gender for attitudes towards the environment, satisfaction with sex life and negative feelings $(p<0.05)$. Men were more satisfied with these dimensions. Age was associated with the environment, ability for daily living, satisfaction with capacity to work and satisfaction with sex life $(p<0.05)$. Older adults between 50 and 59 years performed better in these variables (Table 6). 
Table 6. Report of quality of life of elderly informal workers $(\mathrm{N}=153)$ by gender and age. Manizales, Caldas, 2012-2013.

\begin{tabular}{|c|c|c|c|c|c|c|}
\hline \multirow[b]{2}{*}{ Variable } & \multirow[b]{2}{*}{ 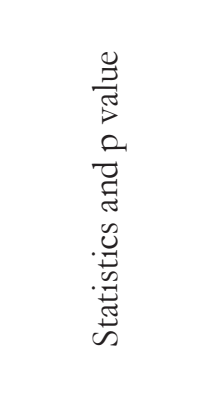 } & \multicolumn{5}{|c|}{ Calidad de vida } \\
\hline & & 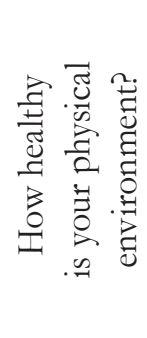 & 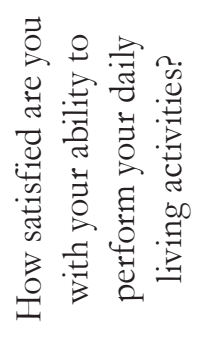 & 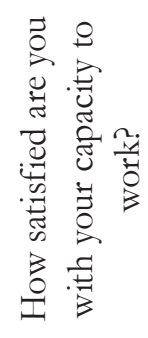 & 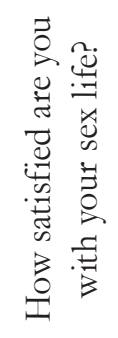 & 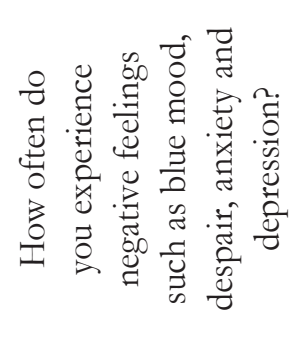 \\
\hline Gender & $X^{2}$ & 5.306 & - & - & 9.607 & 8.326 \\
\hline Female & $p$ value & 0.07 & - & - & 0.008 & 0.016 \\
\hline Male & Phi & 0.186 & - & - & 0.251 & 0.233 \\
\hline Age range & $\mathrm{X}^{2}$ & 14.001 & 16.892 & 13.485 & 18.223 & - \\
\hline $50-59$ & $p$ valor & 0.007 & 0.002 & 0.009 & 0.001 & - \\
\hline $60-69$ & \multirow{2}{*}{$\begin{array}{c}\text { Contingency } \\
\text { coefficient }\end{array}$} & \multirow[b]{2}{*}{0.303} & \multirow{2}{*}{0.332} & \multirow{2}{*}{0.285} & \multirow{2}{*}{0.326} & \multirow{2}{*}{ - } \\
\hline 70 and older & & & & & & \\
\hline
\end{tabular}

\section{DISCUSSION}

A number of studies have explored the quality of life of elderly persons and found common factors with respect to this characteristic in the overall population. Scientific evidence of the relationship between quality of life and informal work among the older population, however, is scarce.

As Manizales has reduced its population growth rate and the population has grown older, there has been a subsequent increase in the dependency ratio, as the income of active workers must support a greater number of older adults. ${ }^{17}$ Regarding sociodemographic characteristics, $69.9 \%$ of the present study were men, in contrast to the study by Estrada et al., ${ }^{18}$ in which $59.4 \%$ were women, and similar to that described in a study by Rivas et al. ${ }^{19}$, where $75.0 \%$ were men. The average age in the present study was $59.4( \pm 7.2)$ years. Estrada et al. ${ }^{18}$ reported an average age of $79.2( \pm 8)$ years. The results were similar in both studies in terms of educational level and social strata. ${ }^{18,19}$

The maximum time spent working in the informal sector in the study population was 59 years, with an average of 23 years, similar to that described by Rivas et al. ${ }^{19}$, where it was clear that the population was dedicated to self-employment, being involved in some kind of modality of informal work from an early age. In terms of matters relating to social security, about $60 \%$ of participants were affiliated to the subsidized health regime and a high percentage had no affiliation with an Office of Risk Management or the pension system, similar to the findings of Rivas et al. ${ }^{19}$ However, in the study by Estrada et al., ${ }^{18} 58.3 \%$ of the participants said they were members of a pension scheme and $46.3 \%$ were affiliated to the subsidized health regime.

$83 \%$ of the older people owned their own businesses. These were related to the sale of sweets, fruits and flowers, activities that require little capital investment. Rivas et al. ${ }^{19}$ found that a high percentage of participants were self-employed.

More than $50.0 \%$ of participants positively assessed their quality of life and health. Studies by Navarro et al. ${ }^{20}$ and Millán, ${ }^{21}$ highlighted three elements that corresponded to the well-being of elderly persons: feeling satisfied with their lives, having the capacity and competence to achieve and maintain control of their environment, and 
conditions of life, fundamental aspects for human self-realization and development.

The older adults did not consider their work place to be very healthy, as the majority of their labor activities took place in public streets and exposed to the dangers of such locations. Zamarrón ${ }^{22}$ stated that the actions of an individual within his or her environment and in that of the workplace are the main factors behind the way in which he or she ages.

Being autonomous was evaluated positively by the participants, and included such aspects such as information for activities of daily living, having money to pay one's day-to-day expenses, the opportunity to take part in leisure, recreation and relaxation activities and the capacity to move from one place to another. In this context, Martí et $a . .^{23}$ found that autonomy is relevant to the individual's evaluation of his or her quality of life, in that it facilitates adaptation to the surrounding environment.

Satisfaction was expressed with activities of daily life, social support and feelings and emotions, which may be explained by the fact that the work of the participants is carried out in a public environment, in contact with the various actors present in such locations including customers, police authorities, passers-by and others working in the same labor conditions, in contrast to other elderly persons who tend to be institutionalized in spaces such as the home and care facilities, restricting their autonomy and in detriment to their social connections and therefore their affective life. Similar findings were reported in a study by Urzúa et al., ${ }^{24}$ in which individuals who were not sick significantly valued social support and their purpose in life. The same situation was also described by Osorio et al. ${ }^{25}$ and by Rivas et al. ${ }^{19}$, who concluded that "social relations and support networks represent the heart of the social interaction of the elderly".

The statistical differences by gender and age in dimensions such as environment, work capacity, ability to perform activities of daily living and sex life were notable; studies such as that by Estrada et al. ${ }^{18}$ found significant differences by gender, age and intimacy while Galán et al. ${ }^{26}$ identified an association between age and environmental quality and satisfaction with life.

Although this study was concluded successfully, it should be noted that the participants agreed to respond to the WHOQOL-BREF quality of life questionnaire on a voluntary basis. However, there were limits for the carrying out of the survey, some interruptions due to the work tasks of the participants, such as attending customers, as well as the conditions of the surrounding environment such as noise and climate.

\section{CONCLUSION}

The quality of life elderly informal workers is viewed favorably and is related to a positive evaluation of health. Work is a factor that stimulates healthy, active and productive aging and the social interaction of many elderly workers favors their quality of life. Furthermore, from the perspective of human development, it gives meaning to the lives of the elderly, allowing them to extend their capacities as an active and productive individual, and contributes to self-realization and social recognition.

These findings can be explored in greater depth by evaluating the effect of the type of work on elderly individuals, through studies with a qualitative focus that lead to the installation of, from a theoretical perspective, public policies that result in relevant and concrete interventions for this growing population group in Latin America.

\section{ACKNOWLEDGEMENTS}

The authors would like to thank those who participated in this study and the Secretaria de Salud Pública de la Alcaldía de Manizales (the Ministry of Public Health of the Municipality of Manizales), for facilitating access to the database that allowed the sample to be calculated and participants to be selected; and the Vicerrectoría de investigaciones y Postgrados de la Universidad de Caldas (the Vice Chancellor of Research and Postgraduate Study of the University of Caldas) for financial support for this study. 


\section{REFERENCES}

1. Castel R. El ascenso de las incertidumbres: trabajo, protecciones y estatuto del individuo. México: Fondo de Cultura Económica; 2010.

2. De La Garza E. Hacia un concepto ampliado del trabajo, de control, de regulación y de construcción social de la ocupación: los otros trabajos. Rev Iztapalapa 2009;30(66):22-36.

3. Organización Internacional del Trabajo. Declaración de Lima. $18^{a}$ Reunión Regional Americana. Lima: OIT; 2014.

4. Organización Internacional del Trabajo, Programa de Promoción de la Formalización en América Latina y el Caribe. Experiencias recientes de formalización em países de América Latina y El Caribe: notas sobre formalización [internet]. Lima: OIT; 2014 [acesso em 12 nov. 2015]. Disponível em: http://www.ilo. org/wcmsp5/groups/public/---americas/---ro-lima/ documents/publication/wcms_245613.pdf

5. Busso M. Cuando una crisis amenaza: un estudio sobre vivencias y percepciones de artesanos argentinos en períodos de crisis socioeconómicas. Ciênc Soc Unisinos 2011;47(3): 292-9.

6. Salas C. ¿Pequeñas unidades económicas o sector informal?". Cotidiano 1992;8(45):1-13. Gómez A. Análisis socio laboral de los vendedores estacionarios en el sector turístico de Bocagrande de la ciudad de Cartagena. Rev Aglala 2010;1(1):1-17. (Esta ref. Possui 2 artigos distintos. que devem ser desmembrados: 1 : Salas C e 2: Gòmez) Ambos estão normalizados.

7. Osta KM. Desempleo e Informalidad en América Latina: definiendo políticas públicas para Venezuela. Rev Venez Gerenc 2007;12(38):262-78.

8. Guerra AE. Consideraciones teóricas acerca de la economía informal, el Estado y la gerencia [monografía en internet]. Barquisimeto: Universidad Centrooccidental Lisandro Alvarado Biblioteca Virtual de Derecho, Economía y Ciencias Sociales; 2007 [acesso em 23 out. 2013]. Disponível em: http://www.eumed. net/libros/2009a/517/Informalidad $\% 20$ para $\% 201 \mathrm{a} \% 20$ Organizacion $\% 20$ Internacional $\% 20 \mathrm{del} \% 20$ Trabajo.htm
9. Arias S, Bernardini D. Retos económicos del envejecimiento. Voces fénix [Internet] 2014 [acesso em 13 nov. 2015];1(36):126-31. Disponível em: http:// www.vocesenelfenix.com/sites/default/files/numero_ pdf/fenix36\%20baja.pdf

10. Orley J, Saxena S. What Quality of Life? World Health Forum 1996;17:354-5. Preparado em nome da WHOQOL GROUP.

11. Dulcey-Ruiz E. Calidad de vida y derecho al reconocimiento en la vejez. Oñati Socio-Legal Ser [Internet] 2011 [acesso em 23 jan. 2014];1(8):9-11. Disponível em: http://opo.iisj.net/index.php/osls/ article/view/86

12. Estrada A, Cardona D, Segura AM, Chavarriaga LM, Ordóñez J, Osorio JJ. Calidad de vida de los adultos mayores de Medellín. Biomédica 2011;31:492-502.

13. Miralles I. Envejecimiento productivo: las contribuciones de las personas mayores desde la cotidianidad. Trab soc 2011;15(16):137-61.

14. Instituto de Mayores y Servicios Sociales. Envejecimiento activo: Libro blanco [Internet]. Madrid: IMSERSO; 2011 [acesso em 13 nov. 2015]. Disponível em: http://www.imserso. es/InterPresent1/groups/imserso/documents/ binario/8088_8089libroblancoenv.pdf

15. República de Colombia. Ministerio de Protección Social. Política Nacional de Envejecimiento y Vejez 2007-2019. [Local desconhecido]: Ministerio de Protección Social; 2007 [acesso em 23 Jan. 2014]. Disponível em: http://www.minsalud. gov.co/Documentos $\% 20 y \% 20$ Publicaciones/ POL $\%$ C3\%8DTICA \%20NACIONAL $\% 20 D E \% 20$ ENVEJECIMIENTO\%20Y\%20VEJEZ.pdf

16. World Health Organization. WHOQOL-BREF: Introduction, administration, scoring and generic version of the assessment. Field Trial Version December 1996. Geneva: WHO; 1996 [acesso em 6 jul. 2013]. Disponible en: http://www.who.int/ mental_health/media/en/76.pdf 
17. Alcaldía de Manizales. Plan de desarrollo 20122015 "Gobierno en la Calle" [Internet]. Minizales: [publicador desconhecido]; 2012 [citado 4 nov. 2015]. Disponível em: http://www.manizales.gov.co/ RecursosAlcaldia/201505052131055709.pdf

18. Estrada A, Cardona D, Segura AM, Chavarriaga, LM, Ordóñez J, Osorio JJ. Calidad de vida de los adultos mayores de Medellín. Biomédica 2011;31(4): 492-502.

19. Romero AV, Rivas AC, Vásquez M, Ramos de la Cruz E, González T, Acosta DP. Calidad de vida del adulto mayor inscrito en los comedores del programa nacional de alimentación para el adulto mayor Juan Luis Londoño De La Cuesta ICBF, 2010-II. DUAZARY [Internet] 2013 [acesso em 22 jan 2014];10(1):26-32. Disponível em: http://investigacion.unimagdalena.edu.co/revistas/ index.php/duazary/article/viewFile/203/181

20. Navarro E, Meléndez JC, Tomás JM. Relaciones entre variables físicas y de bienestar en la calidad de vida de las personas mayores. Gerokomos [Internet] 2008 [acesso em 30 jan. 2014];19(2):17-21. Disponível em:http://scielo.isciii.es/scielo.php?script $=$ sci_ arttext\&pid=S1134-928X2008000200002\&lng=es.
21. Millán JC. Envejecimiento y calidad de vida. Rev Galega Econ [Internet] 2011 [acesso em 30 jan. 2014];20(supl):1-13. Disponible en: https://www.usc. es/econo/RGE/Vol20_ex/castelan/art5c.pdf

22. Zamarrón MD. Envejecimiento activo: un reto individual y social. Soc Utopía 2013;(41):449-63.

23. Martí J, Martínez F, Martí M, Marí R. Responsabilidad Social Universitaria: acción aplicada de valoración del bienestar psicológico en personas adultas mayores institucionalizadas. Polis 2007;18:1-13.

24. Urzúa A, Bravo M, Ogalde M, Vargas C. Factores vinculados a la calidad de vida en la adultez mayor. Rev Med Chile 2011;139(8):1006-14.

25. Osorio P, Torrejón MJ, Anigstein MS. Calidad de vida en personas mayores en Chile. Rev Mad, Univ Chile 2011;24:61-75.

26. Galán JD, García MD, Betancort M. Calidad de vida en personas mayores y su relación con el sentido de vida. Inf Invest Educ 2011;25(1):35-50. 